\title{
HIV+ Status and Cervical Cancer: Cytological Aspects of Cervical Smear in Cameroon Setting
}

\author{
Essome Henri ${ }^{1,2}{ }^{*}$, Engbang Jean Paul ${ }^{1,2}$, Ekono Michel Roger ${ }^{1}$, Nana Njamen Theophile ${ }^{3}$, \\ Mve Koh Valere4, Boten Merlin',2, Tocki Toutou Grâce², Fewou Amadou ${ }^{4}$ \\ ${ }^{1}$ Faculty of Medicine and Pharmaceutical Sciences, University of Douala, Douala, Cameroon \\ ${ }^{2}$ Laquintinie Hospital, Douala, Cameroon \\ ${ }^{3}$ Faculty of Health Sciences, University of Buea, Buea, Cameroon \\ ${ }^{4}$ Faculty of Medicine and Biomedical Sciences, University of Yaounde 1, Yaoundé, Cameroon \\ Email: *essometocky@yahoo.com
}

How to cite this paper: Henri, E., Paul, E.J., Roger, E.M., Theophile, N.N., Valere, M.K., Merlin, B., Grâce, T.T. and Amadou, F. (2020) HIV+ Status and Cervical Cancer: Cytological Aspects of Cervical Smear in Cameroon Setting. Open Journal of Obstetrics and Gynecology, 10, 76-84.

https://doi.org/10.4236/ojog.2020.101007

Received: November 16, 2019

Accepted: January 5, 2020

Published: January 8, 2020

Copyright $\odot 2020$ by author(s) and Scientific Research Publishing Inc. This work is licensed under the Creative Commons Attribution International License (CC BY 4.0).

http://creativecommons.org/licenses/by/4.0/

\begin{abstract}
Introduction: The early detection of precancerous lesions being very important for the preventive management of cervical cancer, we felt it was important to identify these lesions on potential backgrounds including HIV-positive (HIV+) women to suggest control strategies of cervical cancer in Cameroon. Objective: To determine the prevalence of precancerous lesions in women infected with the human immunodeficiency virus (HIV), to investigate the determinants of the HIV and cervical cancer association, and to make recommendations regarding cervical cancer screening in these patients of the city of Douala. Methodology: Our study was a case-control cross-sectional study from July 2017 to December 2017 (6 months) including 108 women among which 34 HIV+ matched with 74 HIV-. HIV serology was done using the complete HIV enzygnost test. Cervical smears for cytological lesions were fixed to the cyto-fixator and then stained by the Papanicolaou technique and read under an optical microscope. The cervical smear slides for viral excretion were fixed with a methanol-acetone mixture of equal volume; HPV testing was done by the indirect immune-peroxidase technique using P16 protein. The excretion of HSV type $1 \& 2$ was investigated by the indirect immunofluorescence technique using the Simplex Virus type $1 \& 2$ Rabbit anti-Herpes from DAKO (France). Results: The two groups of women were compared with the chi square test with a significance threshold of $\mathrm{P}<0.05$. The average age was 40.07 with extremes of 21 and 71 years and a standard deviation of 9.99. Of the $34 \mathrm{HIV}+$ patients, 23 had an abnormal cervix compared to 36 cases of abnormal cervix among HIV- with a statistically significant difference $(\mathrm{P}=0.006649) .12$ cases of dysplasia were observed in the 34 HIV+ women and distributed as follows: $0 \%$ of mild dysplasia, $18.92 \%$ of
\end{abstract}


moderate dysplasia and $13.51 \%$ of severe dysplasia. In HIV- women we detected 6 cases of dysplasia including 1.35\% of mild dysplasia, $4.05 \%$ of moderate dysplasia and $2.70 \%$ of severe dysplasia. Regarding HPV infection, we observed 21 cases of HPV among 34 HIV+ women (61.76\%) against 23 cases in HIV - women (31.08\%); we did not detect any cases of HSV. Conclusion: The prevalence of precancerous lesions remains high in HIV+ women, hence the need to include routine screening for precancerous lesions in all HIV+ women at all ages, as well as the routine search for HPV excretion in all those with cellular dysplasia.

\section{Keywords}

HIV, HPV, Dysplasia, Cervical Cancer

\section{Introduction}

Invasive cervical cancer is a pathology of viral aetiology, which generally takes more than 25 years to develop, from primary infection with an oncogenic papillomavirus of genital tropism to the different grades of histological pre-cancerous lesions, reflecting the persistence of infection to invasive cancer. For each grade of precancerous cervical lesion (CIN1, CIN2 or CIN3), there is a probability of regression to a normal epithelium, and a probability of persistence or progression to a more advanced stage [1]. Once mild dysplasia is identified, it usually takes between 10 and 20 years before invasive cervical cancer develops. $60 \%$ or more of mild dysplasia regress spontaneously and only $10 \%$ progress to moderate or severe dysplasia within 2 to 4 years.

In some cases, moderate or severe dysplasia may occur without going through the detectable stage of mild dysplasia.

Severe dysplasia is more likely to progress to invasive cancer, but less than $50 \%$ of cases progress to invasive cancer. This percentage is even lower among young women. In the early 1990s, it appeared that precancerous lesions and invasive cervical cancer were more common and more rapid in HIV infection [2]. It is estimated that the incidence of invasive cervical cancer is about 5 to 15 times higher in HIV- infected women than in the general population [3].

A prospective study conducted in 15 European countries between 1993 and 1999 among women aged 20 to 49 years shows that invasive cancer of the cervix is the AIDS classifying event in $2.5 \%$ of cases [4].

The incidence of cervical cancer remains high in women with HIV infection. In France, the incidence of cervical cancer increased from 33.7 per 100,000 woman-years (95\% CI: 14.6 - 52.7) in 1996 to 21.5 per 100,000 women-years (95\% CI: 6.6 - 36.5) in 2011 [5].

In the US, the standardized incidence rate of cervical cancer in HIV- infected women is 2.9 ( $95 \%$ CI 1.9 - 4.2), significantly higher compared to HIV- women.

However, the incidence is similar in HIV- infected women who are regularly 
screened for smear and treated for precancerous lesions and in HIV- women, which favors a positive impact of screening on the occurrence of cervical cancer [6].

In contrast, the effect of antiviral treatment on the incidence of this cancer remains controversial. Indeed, the stability of the cervical cancer rate in the US between 1992 and 2003 suggests a weak effect of anti-retroviral treatment, whereas, on the other hand, French data shows a risk of cancer twice as low in women under treatment for more than 6 months [6] [7].

The proportion of women infected with HIV is currently increasing and their survival is prolonged due to anti-retroviral drugs as well as efforts to prevent opportunistic infections by anti-infectious therapies [8]. However, we observe the emergence of cervical intraepithelial pathology that can lead to invasive carcinoma.

In Cameroon, a descriptive and retrospective study carried out between October 1992 and June 1995 in the city of Yaoundé among 65 HIV+ women and 50 HIV- women revealed 95\% inflammatory smear, 1.5\% low-grade squamous intraepithelial lesions in HIV+ women and $70 \%$ of inflammatory smears, $4 \%$ of low-grade squamous intraepithelial lesions in HIV- women with a difference not statistically significant [8].

However, HIV+ women appear to have pre-cancerous cervical lesions more frequently than HIV-, thus predisposing them to cervical cancer in the longer term [8]. The absence of a publication in Cameroon on the immune-histochemical contribution in the detection of precancerous lesions on cervical smears justifies the present study whose objective was to evaluate cervical smear abnormalities in HIV + women as well as their prevalence, as well as the prevalence of cervical HPV 16 and HSV 1 \& 2 infection in both HIV+ women and HIV- women.

\section{Methodology}

Type of study: This was a cross-sectional analytical case-control study from July 2017 to December 2017 (6 months).

Patients and methods: This study included all women present in the day hospital and anatomo-pathology departments of Laquintinie Hospital during the study period and having agreed to participate following informed consent. Sampling was consecutive. Each participant's clinical observation was done but variables of interest retained for this study were age, occupation, clinical variables (history of metrorrhagia and macroscopical aspect of the cervix), biological and cytological variables.

Excluded were all those who expressed the refusal as well as those who were under anti-retroviral treatment.

The cervical smears were performed in the anotomo-pathology department of the Laquintinie hospital after spreading on seven different slides, two of which were fixed with the hairspray for Papanicolaou staining, three fixed with the acetone-methanol mixture of equal volume for the HPV search and two fixed on 
simple methanol for HSV research. The search for HSV type 1 \& 2 excretion was done using the indirect immunofluorescence technique using DAKO's "Rabbit Anti-Herpes simplex virus type 1 and type 2".

The search for excretion of the human papilloma virus was by the indirect immuno-peroxidase technique using the antibodies directed against the papilloma virus protein P16ink4a.

The slides used for viral research were stored for 10 minutes in the refrigerator at $4^{\circ} \mathrm{C}$ and then dried in the open air and wrapped in aluminum foil, then transported to the virology laboratory of the Faculty of Medicine and Biomedical Sciences of Yaoundé where viral research was done.

HIV serologies were performed at the day hospital of the Laquintinie hospital using Enzygnost HIV Integral (enzyme immunoassay) test, simultaneously testing for HIV antigens and anti-HIV antibodies.

Both groups of women were compared with the chi square test with a significance level of $\mathrm{P}<0.05$.

\section{Results}

Our study population consisted of 108 women including $34 \mathrm{HIV}+$ women (31.50\%) matched to $74 \mathrm{HIV}$ - women $(68.50 \%)$; a ratio of $1 \mathrm{HIV}+$ woman to $2.17 \mathrm{HIV}$ - women.

The natural history of cervical cancer takes shape with the precocity of unprotected sex in an environment of social precariousness exposing to a multi sexual partnership and therefore to sexually transmitted infections including HPV.

These variables commonly associated with HPV infection have not been studied in our series; however, the age and occupation variables were invariably distributed in both groups.

The mean age was 40.07 with extremes of 21 and 71 years and a standard deviation of 9.99 with a peak in the $31-40$ age group in both groups [Table 1]; housewives were in majority [Table 2].

\section{Socio-Demographic Characteristics}

The metrorrhagia variable was more frequent in HIV- women $(29.73 \%)$ than in HIV+ women (20.59\%), with a statistically insignificant difference $(\mathrm{P}=$ 0.8336) [Table 3].

Macroscopic cervical lesions included eight (8) variables including abnormal staining, ulceration, budding, contact hemorrhage, condyloma, cervical blocking mass, necrosis, loss of substance.

The data analysis revealed that abnormal cervical coloration, contact hemorrhage, and condyloma were the most common lesions in HIV+ women with a statistically significant difference compared to the control group (HIV-); concerning the coloration of the cervix $(33.82 \%$ against $42.68 \% \mathrm{P}=0.006549)$ the contact hemorrhage (33.82\% against $24.39 \% \mathrm{P}=0.00666)$ and the condylomas (8.82\% against $2.44 \% \mathrm{P}=0.00588$ ) [Table 4]. 
Table 1. Population distribution by age range.

\begin{tabular}{ccccccc}
\hline HIV serology & \multicolumn{2}{c}{ HIV+ } & \multicolumn{2}{c}{ HIV- } & \multicolumn{2}{c}{ Total } \\
\hline Age (years) & N & $\%$ & N & $\%$ & N & $\%$ \\
\hline $21-30$ & 8 & 23.53 & 8 & 10.81 & 16 & 14.81 \\
$31-40$ & 14 & 41.18 & 32 & 43.24 & 46 & 42.59 \\
$41-50$ & 8 & 23.53 & 25 & 33.78 & 33 & 30.56 \\
$51-60$ & 2 & 5.88 & 5 & 6.76 & 7 & 6.48 \\
$61-70$ & 1 & 2.94 & 4 & 5.41 & 5 & 4.63 \\
$>70$ & 1 & 2.94 & 0 & 0 & 1 & 0.93 \\
Total & 34 & 100 & 74 & 100 & 108 & 100 \\
\hline
\end{tabular}

Table 2. Population distribution by occupation.

\begin{tabular}{ccccccc}
\hline HIV serology & \multicolumn{2}{c}{ HIV+ } & \multicolumn{2}{c}{ HIV- } & \multicolumn{2}{c}{ Total } \\
\hline Occupation & N & $\%$ & N & $\%$ & N & $\%$ \\
\hline Student & 0 & 0.00 & 1 & 1.35 & 1 & 0.93 \\
Housewife & 14 & 41.18 & 33 & 44.59 & 47 & 43.52 \\
Retailer & 5 & 14.71 & 3 & 4.05 & 8 & 7.41 \\
Salaried & 8 & 23.53 & 25 & 33.78 & 33 & 30.56 \\
Hair dresser & 1 & 2.94 & 0 & 0.00 & 1 & 0.93 \\
Tailor & 4 & 11.76 & 4 & 5.41 & 8 & 7.41 \\
Farmer & 0 & 0.00 & 4 & 5.41 & 4 & 3.70 \\
Health personnel & 2 & 5.88 & 4 & 5.41 & 6 & 5.56 \\
Total & 34 & 100 & 74 & 100 & 108 & 100 \\
\hline
\end{tabular}

Table 3. Population distribution according to history of metrorrhagia.

\begin{tabular}{ccccccc}
\hline HIV serology & \multicolumn{2}{c}{ HIV+ } & \multicolumn{2}{c}{ HIV- } & \multicolumn{2}{c}{ Total } \\
\hline Metrorrhagia & N & $\%$ & N & $\%$ & N & $\%$ \\
\hline Yes & 7 & 20.59 & 22 & 29.73 & 29 & 26.85 \\
No & 27 & 79.41 & 52 & 70.27 & 79 & 73.15 \\
Total & 34 & 100 & 74 & 100 & 108 & 100 \\
\hline
\end{tabular}

\section{Macroscopic Lesions of the Cervix}

The study of microscopic cervical lesions involved four (4) variables, including inflammation of the cervix, hemorrhage, reshuffling lesions and dysplasia of the cervix.

It showed a high prevalence of metaplastic reshuffling lesions and cervical dysplasia in the HIV + population of $26.47 \%$ versus $6.76 \%(P=0.00461)$ and $35.29 \%$ versus $8.10 \%(\mathrm{P}=0.0004301)$ [Table 5 and Table 6].

\section{Microscopic Lesions of the Cervix}

Research of Viral Markers by Immuno-Histochimics 
Table 4. Summary of macroscopic lesions of the cervix.

\begin{tabular}{cccccccc}
\hline Macroscopiques ducol & \multicolumn{2}{c}{ HIV+ } & \multicolumn{2}{c}{ HIV- } & Total & X2 & P \\
\hline & N & $\%$ & N & $\%$ & N & & \\
\hline Abnormal cervical coloration & 23 & 33.82 & 35 & 42.68 & 58 & 3.39 & 0.006549 \\
Ulceration & 5 & 7.35 & 5 & 6.10 & 10 & 1.75 & 0.1856 \\
Budding & 10 & 14.72 & 18 & 21.95 & 28 & 0.31 & 0.5752 \\
Contact hemorrhage & 23 & 33.82 & 20 & 24.40 & 43 & 6.85 & 0.00666 \\
Condyloma & 6 & 8.82 & 2 & 2.44 & 8 & 7.59 & 0.00588 \\
Cervical blocking mass & 1 & 1.47 & 0 & 0 & 1 & 2.2 & 0.13829 \\
Necrosis & 0 & 0 & 1 & 1.21 & 1 & 0.46 & 0.49587 \\
Loss of substance & 0 & 0 & 1 & 1.21 & 1 & 0.46 & 0.49587 \\
TOTAL & 68 & 100 & 82 & 100 & 150 & & \\
\hline
\end{tabular}

Table 5. Frequency of reshuffling metaplastic lesions of the cervix according to HIV status.

\begin{tabular}{cccccccc}
\hline HIV serology & \multicolumn{2}{c}{ HIV+ } & \multicolumn{2}{c}{ HIV- } & \multicolumn{2}{c}{ Total } & p-value \\
\hline Metaplastic reshuffling & N & $\%$ & N & $\%$ & N & $\%$ & \\
\hline Presence of metaplastic reshuffling & 9 & 26.47 & 5 & 6.76 & 14 & 12.96 & 0.00461 \\
Absence of metaplastic reshuffling & 25 & 73.53 & 69 & 93.24 & 94 & 87.04 & \\
Total & 34 & 100 & 74 & 100 & 108 & 100 \\
\hline
\end{tabular}

Table 6. Frequency of dysplastic lesions of the cervix according to HIV status.

\begin{tabular}{cccccccc}
\hline HIV serology & \multicolumn{2}{c}{ HIV+ } & \multicolumn{2}{c}{ HIV } & \multicolumn{2}{c}{ Total } & p-value \\
\hline Dysplastic lesions & $\mathrm{N}$ & $\%$ & $\mathrm{~N}$ & & $\mathrm{~N}$ & $\%$ & \\
\hline Absence of dysplasia & 22 & 64.70 & 68 & 91.89 & 90 & 83.33 & \\
Mild dysplasia & 0 & 0.00 & 1 & 1.35 & 1 & 0.93 & \\
Moderate dysplasia & 7 & 20.58 & 3 & 4.05 & 10 & 9.26 & 0.0004301 \\
Severe dysplasia & 5 & 14.70 & 2 & 2.7 & 7 & 6.48 & \\
Total & 34 & 100 & 74 & 100 & 108 & 100 & \\
\hline
\end{tabular}

In our series of 108 women, we found many more HPV markers in HIV+ women than in HIV- women; 21 out of $34 \mathrm{HIV}+(61.76 \%)$ versus 23 out of 74 HIV- (31.08\%) ( $\mathrm{P}=0.0025775)$ [Table 7].

In this study, we did not have a positive result for the search for Herpes Simplex Virus type 1 and 2 excretions by indirect immunofluorescence.

\section{Discussion}

The aim of this study was to contribute to research and documentation on cervical cancer in Cameroonian women infected with HIV.

The results obtained were discussed based on clinical, cytological and immunohistochemical variables. 
Table 7. Research of HPV in both groups.

\begin{tabular}{cccc}
\hline Excretion of HPV & HIV $+(\mathrm{N}=\mathbf{3 4})$ & HIV- $(\mathrm{N}=74)$ & P-value \\
\hline Number & 21 & 23 & 0.0025775 \\
Percentage & 61.76 & 31.08 & \\
\hline
\end{tabular}

\section{Epidemiology}

Thirty-four (34) out of 108 subjects in our sample were HIV positive; a prevalence of $31.50 \%$.

This prevalence is high compared to data from the literature; this is because some recruited patients were known HIV+ from the onset.

Clinical, cytological and immunohistochemical data

Regarding the appearance of the cervix, 33.82\% of HIV+ subjects had an abnormal cervical coloration compared to $42.68 \%$ in HIV- subjects with a statistically significant difference $(\mathrm{P}=0.006549)$. The same observation is reported by other authors including MBAKOP [8].

Contact hemorrhage was found in $33.82 \%$ of HIV+ subjects against $24.39 \%$ in HIV - subjects; the HIV+ status in this light had an influence on the occurrence of contact hemorrhages at the cervix $(P=0.00666)$; this observation agrees with that of other authors [9] [10] [11].

Regarding growths, $14.72 \%$ of HIV+ women had a budding cervix against $21.95 \%$ among HIV - with however a non-significant statistical difference; which suggests that other determinants apart from HIV infection would participate in the budding of the cervix.

However, $8.82 \%$ of HIV+ subjects had flat condyloma versus $2.44 \%$ in the matched group with a statistically significant difference $(P=0.00588)$; this high prevalence attests to the correlation between flat condyloma and HIV+ status [10] [11].

The HIV+ status did not have a significant influence on the occurrence of cervical ulcerations (7.35\% in HIV+ versus $6.10 \%$ in $\mathrm{HIV}-, \mathrm{P}=0.1856$ ).

The mass obstructing the cervix, the necrosis and the loss of substances found in this study do not seem to us to be related to the serological status.

Metaplastic shuffling as well as dysplasia were strongly correlated here to $\mathrm{HIV}+$ status; this finding agrees with the data of the literature [10] [11].

The same is true for HPV infection, where $61.76 \%$ of HPV 16 positive serology is found in HIV+ women (21 cases) compared to $31.08 \%$ in HIV- (23 cases) women, with a difference statistically significant $(P=0.0025775)$ [9] [10] [11].

On the other hand, the excretion of HSV $1 \& 2$ is not found in this study and does not seem to correlate with the HIV serological profile.

But our study reveals a strong correlation between papilloma and the occurrence of cervical dysplasia as reported by other authors [9] [10] [11] [12] [13].

The limits of our study

This study, however, has limitations because of the choice of the site (Laquintinie hospital), whose users mainly come from poor social strata and the pay- 
ment nature of the basic analyzes like the cervical smear which limited the sample (108). All of which does not allow absolute conclusions. The same is true for the technique used for viral excretion (immunofluorescence).

\section{Conclusions}

The results of our study confirm the data of the literature making HIV+ status the determining factor in the emergence of cervical dysplasia.

As such we suggest the following:

- To include systematic screening for cervical lesions in all HIV+ women regardless of age;

- To systematically search for HPV excretion in all HIV+ women with cellular dysplasia;

- To intensify educational activities on HIV/AIDS and cervical cancer in Cameroon;

- To systematically put HIV+ women with cellular dysplasia under anti-retroviral therapy even if the CD4 count is normal and the viral load undetectable.

\section{Acknowledgements}

All authors thank the administration of the hospital laquintinie and the staff of the departments of gynecology and anatomo-pathological for all the facilities granted to them during this study.

\section{Data from the Literature}

HIV infection is a determining factor in the emergence of viral HPV-induced cervical dysplasia

\section{The Contribution of Our Study to Research}

Our study confirms data from the literature and suggests screening strategies for dysplasias in HIV+ women.

\section{Contribution of the Authors}

ESSOME collected the data, designed the manuscript and wrote the article. ENGBANG, MVE KOH, NANA, BOTEN and TOCKI read and corrected the article, FEWOU supervised the writing, corrected the article and validated the final version

\section{Conflicts of Interest}

The authors declare that they have no conflict of interest.

\section{References}

[1] Radhia, H. (2017) Frottis cervico utérin: La prise en charge des patientes vivant avec le vih est-elle optimale sur le territoire de la COREVIH Ile de France Nord? Thèse 
de médecine université, Paris, 7.

[2] Carlos Véo, A.R., et al. (2008) Study on the Prevalence of Human Papillomavirus in the Anal Canal of Women with Cervical Intraepithelial Neoplasia Grade III. European Journal of Obstetrics, Gynecology, and Reproductive Biology, 140, 103-107. https://doi.org/10.1016/j.ejogrb.2008.02.024

[3] Maiman, M., et al. (1990) Human Immunodeficiency Virus Infection and Cervical Neoplasia. Gynecologic Oncology, 38, 377-382. https://doi.org/10.1016/0090-8258(90)90077-X

[4] Gallagher, et al. (2001) Cancer Incidence in New York State Acquired Immunodeficiency Syndrome Patients. American Journal of Epidemiology, 154, 544-556. https://doi.org/10.1093/aje/154.6.544

[5] Serraino, D., et al. (2002) Invasive Cervical Cancer as an AIDS-Defining Illness in Europe. AIDS, 16, 781-786. https://doi.org/10.1097/00002030-200203290-00014

[6] Stewart Massad, L., et al. (2009) Long-Term Incidence of Cervical Cancer in Women with Human Immunodeficiency Virus. Cancer, 115, 524-530. https://doi.org/10.1002/cncr.24067

[7] Guiguet, M., et al. (2009) Effect of Immunodeficiency, HIV Viral Load, and Antiretroviral Therapy on the Risk of Individual Malignancies (FHDH-ANRS CO4): A Prospective Cohort Study. The Lancet Oncology, 10, 1152-1159. https://doi.org/10.1016/S1470-2045(09)70282-7

[8] Mbakop, A., Zekeng, L., Mbassi, J.R. and Essimbi, F. (1996) Aspects cytologiques du frottis cervical en microscopie optique chez les femmes séropositives à Yaoundé-Cameroun (Afrique Centrale). Archives D'anatomie et de Cytologie Pathologiques, 44, 5-6.

[9] Criton, C. (2010) Lésions précancéreuses du col utérin et infection à VIH et al. Obstétrique \& Fertilité, 38, 199-204.

[10] Abraham, A.G., et al. (2013) Invasive Cervical Cancer Risk among HIV-Infected Women: A North American Multi-Cohort Collaboration Prospective Study. Journal of Acquired Immune Deficiency Syndromes, 62, 405-413.

[11] Strickler, H.D., Burk, R.D., Fazzari, M., et al. (2005) Natural History and Possible Reactivation of Human Papillomavirus in Human Immunodeficiency Virus-Seropositive Women. Journal of the National Cancer Institute, 97, 577-586. https://doi.org/10.1093/jnci/dji073

[12] Ahdieh, L., Munoz, A., Vlahov, D., et al. (2000) Cervical Neoplasia and Repeated Positive of Human Papillomavirus Infection in Human Immunodeficiency Virus-Seropositive and -Seronegative Women. American Journal of Epidemiology, 15, 1148-1157. https://doi.org/10.1093/oxfordjournals.aje.a010165

[13] Massad, L.S., Ahdieh, L., Benning, L., et al. (2001) Evolution of Cervical Abnormalities among Women with HIV-I: Evidence from Surveillance Cytology in the Women's Interagency HIV Study. Journal of Acquired Immune Deficiency Syndromes, 27, 432-442. https://doi.org/10.1097/00042560-200108150-00003 